\title{
A Novel Biomedical Blood Cell Recognition Method Based on XAVIS
}

\author{
J. LIU \\ ${ }^{1}$ School of Electronic and Information Engineering, Xi'an Jiaotong University \\ ${ }^{2}$ School of Electrical Engineering, Xi'an Jiaotong University \\ J.Han*, H. Lv \\ School of Electronic and Information Engineering, Xi'an Jiaotong University
}

\begin{abstract}
Computer aided diagnosis method have been more and more widely used in biomedical area. A novel cell particle extraction method based on smooth filtering and mathematical morphology is proposed to detect particles such as white and red blood cells in blood smear images with serious background noise, and it is able to get the total number of white blood cells and their sizes for disease diagnosis. Both methods have been embedded in a machine vision software XAVIS, which can act as an assistance for disease diagnosis.

KEYWORD: Computer aided diagnosis; biomedical particle image; particle recognition; XAVIS
\end{abstract}

\section{INTRODUCTION}

Traditionally, observation on biomedical images, mainly depends on microscope, which assists the doctor to study the properties of the possible disease from the microstructure observation[1]. Biomedical microscopic images have been widely used in the following areas: pathology, immunology, clinical diagnosis and genetics, etc[2]. For example, In clinical application, to analyze the particle image of the patient's blood samples while doing routine blood tests, detection of red blood cells and white blood cells in a certain proportion, the platelet count, average red blood cell volume, average hemoglobin content and average haemoglobin concentration and lymph cell percentage, allows the physician to judge the person's health basically[3]. In deciding whether the body has inflammation, a blood test particle image can also be applicable, to observe whether the number of white blood cells exceed standard amount, or whether the cell shape changes[4][5]. Also in some intestines and stomach and digestive system disease detection, the detection and analysis of particle image of a urine sample will be very helpful for diagnosis.

For these test samples, such as pathological tissue section as well as a variety of body fluids and blood smear, will eventually be processed as a biomedical image with different shapes and distribution of particles. These microscopic image enables physicians observe all kinds of cells in human body, the status of pathogen, the proportion of cells in the image, or the distribution of important components. Combining the testing data generated by biomedical images, with other pathological features, doctors will be able to perform clinical diagnosis correspondingly.

In biomedical imaging aided diagnosis, accurate detecting of the particle image is very important to the final judgment of doctors. Previously, studies on detection of some kinds of particles and basic methods or systems have been developed, such as specific cell count statistics systems[6], automatic segmentation and recognition of dual-core cell microscopic images[7], automatic recognition of bone marrow cell images[8], etc. These testing system has their specific applicability. In this paper, a more general XAVIS software platform for detecting biomedical particle image is presented. In the software, a new evaluation method for particle image detection and distribution is proposed, which is able to classify images with different nonuniformity distribution.

The structure of the paper is as follow. The next section introduces the basic function of XAVIS. Section III presents a novel particle image recognition method, based on image smoothing and of mathematical morphology. And the experimental results and analysis is tested to segment biomedical image particles from the noisy background, which can be seen in Section IV. Finally, the conclusions are presented in Section V.

\section{BASIC FUNCTION OF XAVIS}

Detection of small cell particles under complex background is one of the main problems in biomedical imaging area, the proposed recognition method is mainly based on smooth filtering, 
mathematical morphology algorithms and the XAVIS architecture.

XAVIS software is developed by the authors and have registered the national software copyright of China (No. 2007SR20093). XAVIS configuration software, is a universal machine vision software, which provides file operations, variable operation, control statements, the graph mark, threshold segmentation, image transformation, image filtering, edge detection, morphological processing, straight side processing, the wavelet transform, image measurement, image matching, image fusion, image stitching, barcode recognition, character recognition, and over 280 image processing functions embedded as its library functions.

\section{BLOOD CELL IMAGE RECOGNITION METHOD}

\subsection{Smooth filtering}

The main purpose of image smooth filtering is to reduce the noise of the original image, which may come from many sources, such as external electromagnetic wave interference into the internal system through the power source[9], camera thermal noise, electrical mechanical movement induced shaking noise inside the system, etc. Two smoothing techniques can be used to reduce noise, in spatial domain or in frequency domain[10]. As for the frequency domain processing method, it basically uses a low-pass filter technology, which will require causes of noise, and the distribution characteristics and its relationship with the image signal. While the spatial domain processing method is mainly based on mean or the median values of the pixels[10]. The average neighborhood method of the space domain is used in this paper for image smoothing.

The average neighborhood method is a local spatial domain processing method, a neighboring set $S$ is constructed to each pixel of original image $f(x, y)$ with noise, then the grayscale average value of all the pixel inside $S$ is calculated, as the pixel value of image $g(x, y)$ after spatial domain average procession:

$$
g(x, y)=\frac{1}{M} \sum_{(x, y) \in S} f(x, y)
$$

where: $f(x, y)$ is an $N \times N$ matrix with $x, y=0,1, \ldots, N-1$, and $S$ is a set of all the points with the center $(x, y), M$ is the pixel numbers of set $S$. Typical neighboring set $S$ can be comprised of 4 or 8 pixel points, as shown in the Figure 1.

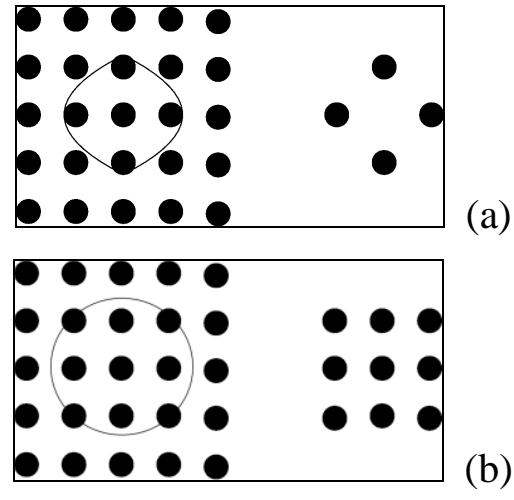

Figure 1 Typical neighboring sets of average neighborhood method: (a) 4 pixel points; (b) 8 pixel points.

The smoothing effect of average neighborhood method is related to the radius of neighboring set $S$. The bigger the radius, the greater the blurring degree of the image. In order to reduce this blurring effect when using larger neighborhood, the smoothing function of Equation(1) can be improved by a new expression as in literature [9]:

$$
g(x, y)=\left\{\begin{array}{cc}
\frac{1}{M} \sum_{(x, y) \in S} f(x, y), & f(x, y)-\frac{1}{M} \sum_{(x, y) \in S} f(x, y) \mid>T(2) \\
f(x, y), & \text { other }
\end{array}\right.
$$

where: $T$ is selected as a nonnegative threshold. For a given radius value, it is able to reduce the blurring effect using the threshold settings. If the average difference between the grayscale value of a certain pixel and average value of its neighborhood is less than $T$, the average value is used in place of the pixel, which is so called the average processing; Otherwise, its original grayscale value is used. This procession is more effective for the inhibition of salt and pepper noise in the image, also has a good protection of small gray level difference and texture information.

\subsection{Mathematical morphology}

The basic principle of using mathematical morphology in image processing is to construct a certain form of shaped structural elements to measure the image, or extract the corresponding shape features for image analysis and recognition. The basic operations of mathematical morphology including Dilation, Corrosion, Opening and Closing, various mathematical morphology algorithm can be developed based on these on the combination and improvement of these operations[10]. For a binary image, it is usually assumed that points with pixel value 1 correspond to the target area of concern, and the points with pixel value 0 correspond to the background area of the image.

\section{(1) Dilation}

Dilation is one of the most basic operators of the morphological operation, its main application in image processing is to extend boundary points, or 
connect close objects. Dilation of set $A$ with set $B$ is defined as $A \oplus B$ :

$$
A \oplus B=\left\{x \mid \hat{B}_{x} \cap A \neq \varnothing\right\}
$$

where: $\oplus$ is the dilation operator, $\hat{B}_{x}$ is the transpose or symmetrical of $B$ and then translated by vector $x$. If $B$ has a center on the origin, then the dilation of $A$ by $B$ can be understood as the locus of the points covered by $B$ when the center of $B$ moves inside $A$. The results of dilation is that when the center of structuring element (or called set) $B$ moves inside the image (or set) $A$, the elements in set $B$ plus the corresponding displacement will be the dilated image. Under the developed XAVIS software framework, the dilation matrix is comprised of $3 \times 3$ structural elements with a radius of 1 :

$$
B=\left(\begin{array}{lll}
0 & 1 & 0 \\
1 & 1 & 1 \\
0 & 1 & 0
\end{array}\right)
$$

\section{(2) Erosion}

Erosion is a dual operator of dilation in mathematical morphology. Its main function is to remove the boundary points of the image, eliminate the objects that are smaller than the structural elements, and separate two objects with weak connection. Erosion of set $A$ by set $B$, is defined as $A$ ! $B$ :

$$
A ! B=\left\{x \mid B_{x} \subseteq A\right\}
$$

where: ! is the erosion operator, $B_{x}$ is the translation of $B$ by vector $x$. When the structuring element $B$ has a center, and this center is located on the origin of coordinate system, then the erosion of $A$ by $B$ can be understood as the locus of points reached by the center of $B$ when $B$ moves inside $A$.

\section{EXPERIMENTAL RESULTS}

\subsection{The flowchart of particle image recognition}

For a particle biomedical image, the procedures of implementing the particle identification algorithm in the framework of XAVIS software is described in Figure 2.

Step1: Smooth filtering of the original image to eliminate background noise, using the average neighborhood method;

Step2: Subtract the original image by the smoothfiltered image, and the difference is the particle area in the original image. Due to the influence of average effect, the particle area will show great variation between the two pictures;

Step3: Perform erosion operation to the subtracted image;
Step4: Perform dilation operation to get clear particles in the image with clear edges;

Step5: Get the number and area of particles using connected domain markers;

Step6: Add the processed image with clear particle edge to the original image, and it will be easy to mark out the exact locations of the particles on the original image.

The image processing results for a typical blood pathological image obtained from [3] are shown in Figure 3.

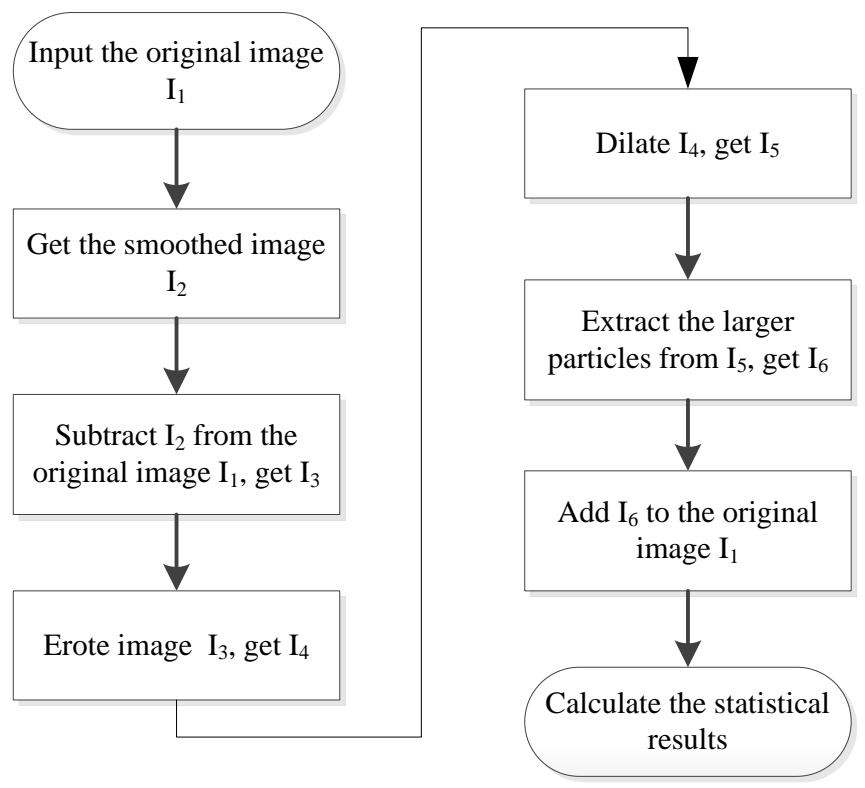

Figure 2 Flowchart of particle image recognition

Using the smooth filtering and mathematical morphological method, it is easy to detect particle edge and find the actual location and area of all particles in a biomedical image, and can detect the particles in the original image. As in the original image of this study, we detect a number of 360 particles, and the total area is 31887 pixels, the area of the largest particle is 432 pixels, while the minimum area is 1 pixel, the size of the average area is 88 pixels. as shown in Figure 3 (a)-(f).

In XAVIS software, larger particles with area of over twice of the average 88 pixels, can be further highlighted in the original image, as shown in Figure 4, which might be considered as potential targets with problems, and the highlighted particles is shown in Figure 4. For this typical pathological image of blood smear samples obtained from [3], because white blood cells is usually larger than red blood cells, if the number of white blood cells within the image is more than healthy situations, which might indicate that the patient have suffered inflammation. Therefore, the proposed particle recognition method is able to provide auxiliary diagnosis, and prompt physicians to pay special attention to larger particles in biomedical images. 


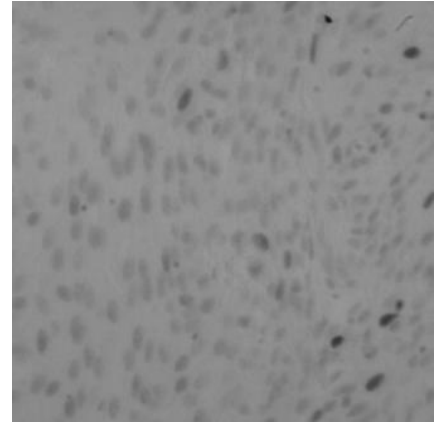

(a)

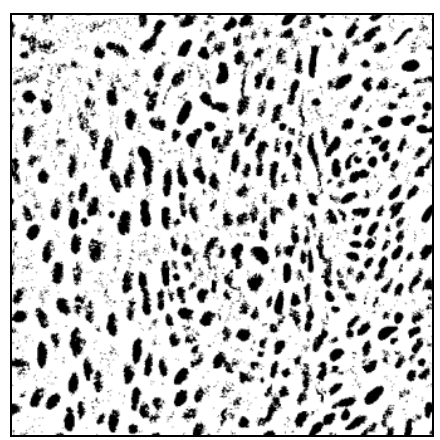

(c)

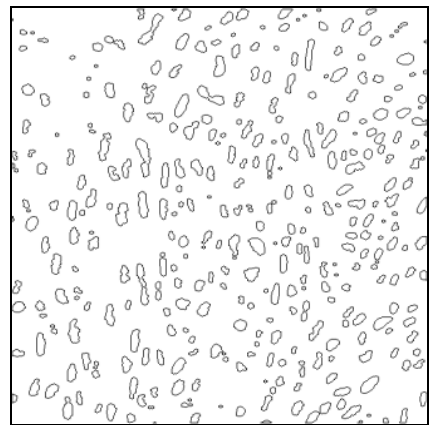

(e)

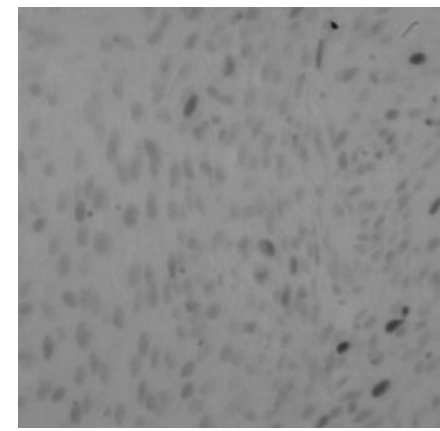

(b)

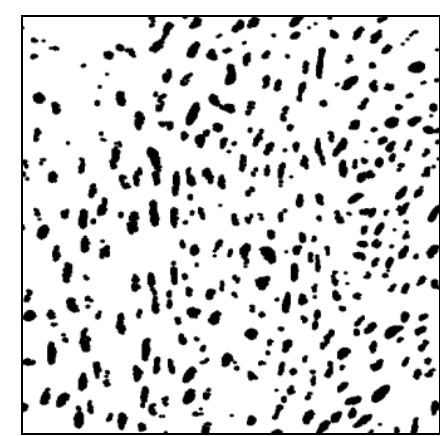

(d)

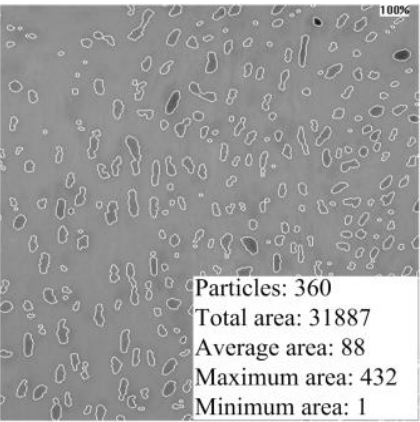

(f)
Figure 3 Procedures and results for particle detection: (a) Original image; (b) After smooth filtering; (c) Subtraction of the original image by filtered image; (d) erosion operation to image(c); (e) particle edge extraction by dilation operation to image(d); (f) statistics of the locations and area of particles marked on the original image.

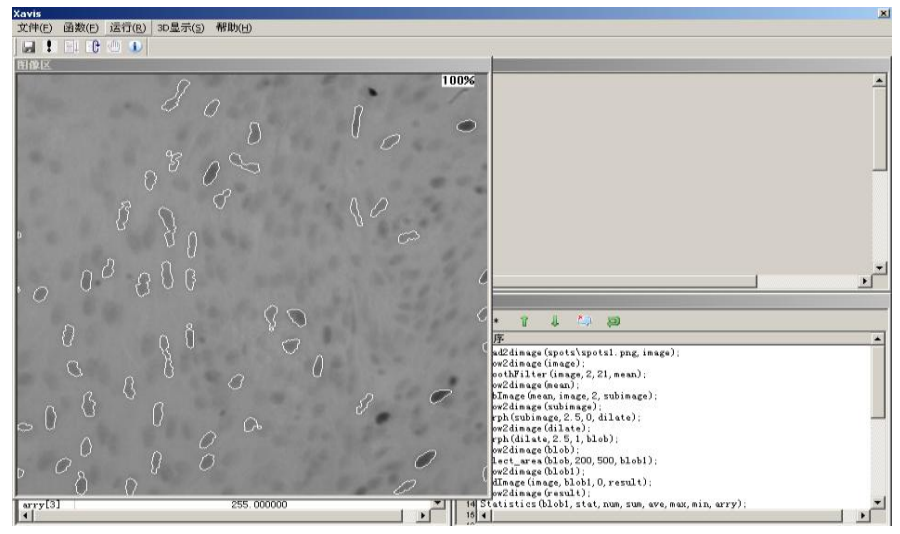

Figure 4 Highlighted larger particle in XAVIS software

\section{CONCLUSION}

Digital image processing methods have been more and more widely used in biomedical diagnosis recently. An particle extraction method based on smooth filtering and mathematical morphology is proposed to extract biomedical blood cell particle images with serious background noise. Connecting area analysis is adopted to get the total number of particles and their sizes, which can provide very valuable information for biomedical use. The proposed method has been embedded in a machine vision software XAVIS, which is able to act as an useful assistance for disease diagnosis.

\section{REFERENCES}

[1] Bushberg J T, Boone J M. The essential physics of medical imaging. Lippincott Williams \& Wilkins, 2011.

[2] Esgiar A N, Naguib R N G, Sharif B S, et al. Microscopic image analysis for quantitative measurement and feature identification of normal and cancerous colonic mucosa. Information Technology in Biomedicine, IEEE Transactions on, 1998, 2(3): 197-203.

[3] Blood Report sample of Armed Police Corps of Shaanxi Province Hospital. 2010.

[4] Giger M, MacMahon H. Computer-aided diagnosis. Radiologic Clinics of North America, 1996, 34: 565-596.

[5] Jiang K, Liao Q M, Dai S Y. A novel white blood cell segmentation scheme using scale-space filtering and watershed clustering. Machine Learning and Cybernetics, 2003 International Conference on. IEEE, 2003, 5: 28202825.

[6] Di Ruberto C, Dempster A, Khan S, et al. Analysis of infected blood cell images using morphological operators. Image and Vision Computing, 2002, 20(2): 133-146.

[7] Sarkar N, Chaudhuri B. An efficient approach to estimate fractal dimension of textural images. Pattern Recognition, 1992, 25(9): 10 35.

[8] Hou Zhenjie, Ma Shuoshi, Pei Xichun, et al . Studies on segmentation and recognition of marrow cells image. In Proceedings of International Symposium on Communications and Information Technology. Beijing: IEEE, 2005. 1263- 1266.

[9] Jain A, Hong L, Pankanti S. Biometric identification. Communications of the ACM, 2000, 43(2): 90-98.

[10]Jain A K. Fundamentals of digital image processing. Prentice-Hall, Inc., 1989. 